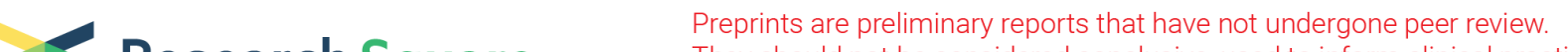

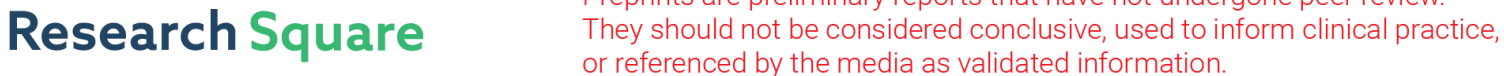

\section{SUM01 modification of IGF-1R combining with SLUG inhibited osteogenic differentiation of PDLSCs stimulated by high glucose}

\section{Rongrong Jiang}

Affiliated Hospital of Nantong University

\section{Miao Wang}

Nantong University

\section{Xiaobo Shen}

Affiliated Hospital of Nantong University

\section{Shuai Huang}

Nantong University

\section{Jianpeng Han}

Affilited Hispital of Nantong University

Lei Li

Nantong University Affiliated Hospital: Affiliated Hospital of Nantong University

\section{Zhiliang Xu}

Affiliated Hospital of Nantong University

Chengfeng Jiang

Affiliated Hospital of Nantong University

\section{Qiao Zhou}

Affiliated Hospital of Nantong University

Xingmei Feng ( $\square$ fengxingmeint@126.com )

Affiliated Hospital of Nantong University https://orcid.org/0000-0001-8046-3336

\section{Research Article}

Keywords: IGF-1R, Differentiation, Periodontal ligament stem cells, SLUG, High glucose

Posted Date: March 24th, 2021

DOl: https://doi.org/10.21203/rs.3.rs-357949/v1

License: (c) (1) This work is licensed under a Creative Commons Attribution 4.0 International License.

Read Full License 


\section{Abstract}

Background: Periodontal disease, an oral disease characterized by loss of alveolar bone and progressive teeth loss, is the sixth major complication of diabetes. It is spreading worldwide as it is difficult to be cured. The insulin-like growth factor 1 receptor (IGF-1R) plays an important role in regulating functional impairment associated with diabetes. However, it is unclear whether IGF-1R expression in periodontal tissue is associated with periodontal bone tissue destruction in diabetic patients. SUMO modification has been reported in various diseases and are associated with an increasing number of biological processes, but previous studies have not focused on diabetic periodontitis.

Methods: Periodontal membrane stem cells (PDLSCs) were isolated and cultured from healthy human obstructed teeth after extraction or adolescent orthodontic subtractive extraction. PDLSCs were cultured with medical $5 \%$ sterile glucose solution formulated as osteogenic differentiation induction solution with different glucose concentrations. The effects of different glucose concentrations on the osteogenic differentiation ability of PDLSCs were investigated at the genetic and cellular levels using staining assay, Western Blot, RT-PCR, Co-IP and cytofluorescence.

Results: We found that SLUG, RUNX2 expression was decreased in PDLSCs cultured in high glucose osteogenic induction solution compared with normal glucose osteogenic induction solution. In addition, the IGF-1R expression levels, osteogenic differentiation and sumoylation of IGF-1R in PDLSCs cultured in high glucose osteogenic induction solution were not consistent with those cultured in normal glucose osteogenic induction solution.

Conclusion: Our data demonstrated that SUMO1 modification of IGF-1R in high glucose environment inhibited osteogenic differentiation of PDLSCs by binding to SLUG, a key factor leading to periodontal bone tissue loss in diabetic patients. Thus we can maximize the control of multiple downstream damage signaling factors and bring new hope for periodontal tissue regeneration in diabetic patients.

\section{Introduction}

Diabetes mellitus (DM) is one of the most prevalent chronic diseases worldwide, and the prevalence of DM in adults is about 10\% [1]. Diabetes mellitus is a metabolic disease characterized by a chronic increase in blood glucose levels, while hyperglycemia is caused by a defect in insulin secretion or impairment of its biological action, or both. The chronic presence of hyperglycemia leads to chronic damage to various tissues, especially the eyes, kidneys, heart, blood vessels, and nerves. Diabetic patients are also more likely to induce chronic periodontal disease, which can lead to tooth loosening, occasional pain and discomfort, impaired masticatory function, and eventually tooth loss $[2,3]$. There are segments of common clinical treatment, such as supragingival scaling, subgingival scraping, local rinsing, loose tooth fixation, etc., which are extremely limited. Diabetic patients would undergo the significantly increased risk of osteoporosis, and the balance between osteoblasts and osteoclasts is disrupted, and osteogenesis is significantly inhibited [4]. All of these tell us that diabetes is a very 
important factor in the loss of periodontal bone tissue. Therefore, it is urgent to find new clinical approaches to treat periodontal regeneration in diabetic patients.

So far, the clinical applications of stem cell therapy are very promising, including dental, craniofacial and orthopedic treatments, especially in bone repair and regenerative therapy [5]. Bone marrow mesenchymal stem cells (MSCs) can differentiate into a variety of bone matrix cells, which have been used to treat osteonecrosis of the femoral head caused by glucocorticoids [6]. However, MSCs exhibit degenerative changes during aging, including unbalanced differentiation and reduced proliferation, resulting in agerelated bone loss [7]. PDLSCs are self-renewing adult multifunctional stem cells of mesodermal origin, derived from periodontal tissue, which can differentiate into osteoblasts, chondrocytes, adipocytes and nerves. Stem cells have been used to treat a variety of diseases, especially in bone defects [8]. PDLSCs are more accessible and have greater activity and proliferation capacity than other MSCs such as BMMSCs and UC-MSCs, therefore it is of great importance to study the specific mechanisms of osteogenic differentiation of PDLSCs, which widely presented in periodontal tissues.

Insulin-like growth factor 1 receptor (IGF-1R) expression was elevated in diabetic patients and was also abnormally elevated in diabetic osteoporosis rat models [9]. IGF-1R is located on the cell membrane and can be activated by insulin-like growth factors, causing phosphorylation of its own tyrosine kinase structural domain and initiating intracellular signaling to regulate cell growth and differentiation [10]. Membrane proteins have multiple functions and play an important role in cell recognition, cell signaling, and transport, and are therefore excellent drug targets. Post-translational modifications (PTMs) are posttranslational chemical modifications of proteins, and PTMs are complex processes that are involved in almost all cellular life processes, which plays an extremely important regulatory role. It has more than 400 kinds, mainly including phosphorylation, glycosylation, acetylation, ubiquitination, sumoylation and some other niche modifications [11]. However, high glucose significantly upregulates the expression of PIASy, SUMO1 and SUMO2/3 in a dose and time dependent manner, inducing IKKY phosphorylation and sumoylization, which then triggers nuclear factor kappa-B (NF-KB) protein signaling [12]. It has been shown that IGF-1R becomes sumoylated by IGF-1 stimulation of the receptor and translocates to the nucleus to act as a transcriptional cofactor and play a biological role [13]. In the serotype model of human corneal epithelial cells, IGF-1R not only mediates the signal transduction on the plasma membrane, but also can be sumoylated through sumo 1, and transported to the nucleus under the action of IGF-1[14]. Therefore, IGF-1R is of great research value in the consideration of the etiology of periodontal disease in diabetic patients.

Snail Family Transcriptional Repressor 2 (SLUG) is a protein-coding gene. This transcription factor is present in stem cells and is essential for osteoblast differentiation and can control cellular osteogenic differentiation. Also, this osteogenic stem cell factor is associated with the transcriptional regulation of stem cell genes and genes activated during osteogenic differentiation [15]. In addition, SLUG binds to the RUNX2 promoter to promote osteogenic differentiation [16]. Its related pathways include direct p53 effector and Hippo signaling pathway. Snail/SLUG can also regulate stem cell function by forming a complex with the transcriptional coactivators YAP and TAZ, inhibiting Hippo pathway-dependent 
regulation of the YAP/TAZ signaling cascade. In turn, the Snail/SLUG axis activates a series of downstream targets of bone formation [17]. In conclusion, these results suggest that the search for the key of upstream and downstream regulation of this transcription factor, SLUG, during osteogenic differentiation of stem cells remains a hot topic of research.

In this study, PDLSCs were isolated and cultured from healthy human obstructed teeth after extraction or adolescent orthodontic subtractive extraction. And the effects of different glucose concentrations on the osteogenic differentiation ability of PDLSCs were investigated at the genetic and cellular levels using staining assay, Western Blot, RT-PCR, Co-IP and cytofluorescence. This study will further enrich the pathogenesis of periodontal diseases caused by diabetic patients and provide a theoretical basis for finding therapeutic targets for periodontal diseases in diabetic patients.

\section{Materials And Methods}

\section{Separation, culture of PDLSCs}

The culture procedure of periodontal stem cells was performed according to the previous research method of our group [18] and was approved by the ethics committee of Nantong University Hospital. Ten orthodontically decompensated premolar and interrupted teeth from patients aged 14-22 years were collected from the outpatient clinic of the Department of General Dentistry, Nantong University Hospital with the informed consent of so adolescent patients. All participants rinsed their teeth with sodium bicarbonate solution before extraction, and immediately after extraction the teeth were placed in phosphate-buffered saline (PBS) containing $100 \mathrm{U} / \mathrm{ml}$ penicillin and $100 \mu \mathrm{g} / \mathrm{ml}$ streptomycin (Invitrogen, Life Technologies, Carlsbad, CA), rinsed twice to remove blood, and treated with a sterile surgical blade in an ultra-clean The periodontal membrane tissue was scraped from the middle third of the tooth surface in the ultra-clean table and digested in $3 \mathrm{mg} / \mathrm{ml}$ type I collagenase (Worthington Biochem, Freehold, $\mathrm{NJ}$ ) and $4 \mathrm{mg} / \mathrm{ml}$ dispase (Roche, Mannheim, Germany) for $30 \mathrm{~min}$. Cell suspensions of periodontal membranes were inoculated into $25 \mathrm{~cm}$ cell culture dishes and suspended in complete medium containing low glucose Dulbecco's modified Eagle's medium (GIBCO BRL, Grand Island, NY) with $10 \%$ fetal bovine serum (FBS), $100 \mathrm{U} / \mathrm{ml}$ penicillin and $100 \mu \mathrm{g} / \mathrm{ml}$ streptomycin. Cells were inoculated in tissue culture dishes and cultured at $37^{\circ} \mathrm{C}$ with $5 \% \mathrm{CO}_{2}$, and the medium was changed every 3 days. When the cells in the culture dish reached $80 \%$, they were cultured in a $1: 3$ ratio of passages. So the experiments were performed using the fourth generation of periodontal stem cells.

Osteogenic differentiation

The osteoblast differentiation process was as described in a previous study by our group [19]. The P4 generation of PDLSCs was used as the study subject and inoculated in 6-well culture plates at a density of 3,000 cells $/ \mathrm{cm}^{2}$, and the osteogenic differentiation induction medium $(50 \mu \mathrm{g} / \mathrm{mL}$ ascorbic acid, $10 \mathrm{mM}$ sodium glycerophosphate, and $1.0 \mu \mathrm{g} / \mathrm{mL}$ dexamethasone) was configured with different glucose concentrations of complete culture medium ( $5.5 \mathrm{mMol} / \mathrm{L}, 11 \mathrm{mMol} / \mathrm{L}, 25 \mathrm{mMol} / \mathrm{L}, 44 \mathrm{mMol} / \mathrm{L}), 11$ 
$\mathrm{mMol} / \mathrm{L}$ and $44 \mathrm{mMol} / \mathrm{L}$ glucose concentrations were configured from L-DMEM with appropriate amount of medical $5 \%$ sterile glucose solution, and differentiation induction cultures were incubated for 7,14 and 21 days, with fluid changes every 1 day.

Alizarin red staining and ALP staining

Cells were co-cultured with osteogenic medium for 21 days and stained to detect the mineralization potential of the cells. The medium in the 6-well culture plate was first discarded, the cells were washed 3 times with appropriate amount of 0.01 M PBS, inverted to drain the remaining water, and the cells were fixed with $4 \%$ paraformaldehyde for 30 minutes. The cells were then washed 3 times with an appropriate amount of $0.01 \mathrm{M}$ PBS and the remaining water was drained. The cells were then incubated with $40 \mathrm{~mm}$ Alizarin Red S solution (Solarbio) for 15 min according to the manufacturer's instructions for Alizarin Red staining and with $40 \mathrm{~mm}$ Alkaline Phosphatase Assay Kit (Beyotime) for $12 \mathrm{~h}$ for Alkaline Phosphatase staining.

Western blot

The cellular protein extraction process was performed as described in a previous study by our group [20]. Cells cultured in osteogenic induction medium with different glucose concentrations were first fully lysed using PMSF solution and RIPA solution (Beyotime), followed by protein collection and centrifugation to extract the supernatant. Membrane, Nuclear and Cytoplasmic Protein Extraction kit (Sangon Biotech, C510002) was used to extract membrane and nuclear proteins from cells cultured in osteogenic medium with different glucose concentrations according to the product instructions, and then the protein concentration was measured by BCA method. The protein concentrations were then measured by BCA to sample the calculated amounts. After $90 \mathrm{~min}$, the PVDF membrane was placed in the closure solution and gently shaken on a shaker for $2 \mathrm{~h}$ at room temperature. The primary antibody was incubated overnight at $4{ }^{\circ} \mathrm{C}$ and the secondary antibody was incubated for $2 \mathrm{~h}$ at room temperature. The following primary antibodies were used: rabbit anti-SLUG(1:1000,Cell Signaling Technology), rabbit anti-RUNX2 (1:1000, Sangon Biotech), rabbit anti-IGF-1R (1:1000, Abcam), rabbit anti-GAPDH (1:1000, Abcam), mouse anti-SUMO1 (1:1000, Santa Cruz),mouse anti-PCNA (1:1000, Santa Cruz), mouse anti-PI3K (1:1000, Santa Cruz), mouse anti-pAKT (1:1000, Santa Cruz), rabbit anti-AKT (1:1000, Proteintech), and rabbit anti-Na ${ }^{+}-$ $\mathrm{K}^{+}$-ATPase (1:1000, Abbkine). The second antibodies were goat-anti-rabbit or goatanti-mouse horseradish peroxidase-conjugated IgG (1:1500, Abcam).

Quantitative real -time reverse transcription-polymerase chain reaction

According to the manufacturer's instructions (Invitrogen), total RNA was extracted from cells using Trizol reagent. Complementary DNA (cDNA) miRNA transcripts were amplified using Maxima ${ }^{\text {TM }} \mathrm{H}$ Minus $\mathrm{cDNA}$ Synthesis Master Mix, with dsDNase (Thermo Fisher Scientific, Waltham, MA). The polymerase chain reaction (PCR) mixture was prepared using LightCycler 480 SYBR Green (Roche Applied Science, Penzberg, Germany). For GADPH, RUNX2 and SLUG detection, cDNA was synthesized using RevertAid RT Reverse Transcription Kit (Thermo Fisher Scientific). AceQ qPCR SYBR Green Master Mix (without ROX) 
(Vazyme) was then used for quantitative PCR of these genes. GAPDH was used for normalization. We use a Light Cycler 480 Real-Time PCR System (Roche Diagnostic, Mannheim, Germany) to test these levels. The primer sequences used in the experiment were as follows:

GAPDH:5'-GAAGGTGAAGGTCGGAGTC-3',5'-GAAGATGGTGATGGGATTTC-3';RUNX2:5'TCAACGATCTGAGATTTGTGGG-3',5'-TCAACGATCTGAGATTTGTGGG-3';SLUG:5'TGTGACAAGGAATATGTGAGCC-3',5'-TGAGCCCTCAGATTTGACCTG-3'.

The ratio obtained by comparing the target gene with the CT value is the relative expression of the gene. Three replicates were set up three times for each experiment.

Co-Immunoprecipitation

Co-immunoprecipitation protocol was performed using a Pierce co-IP kit (Thermo Scientific ${ }^{\text {TM }}$ Pierce ${ }^{\text {TM }}$ Classical magnetic bead immunoprecipitation/immunocoprecipitation Kit, 88804). Incubate the cell lysate with the rabbit anti-IGF-1R (Abcam) for 1-2 hours at room temperature, or overnight at $4{ }^{\circ} \mathrm{C}$. Bind the antigen/antibody complex to the protein $A / G$ magnetic beads for one hour at room temperature. Wash the beads twice with immunoprecipitation lysis/rinse buffer, followed by one wash with pure water. Elute the antigen/antibody complexes. Finally, immunoblotting experiments for immune complexes were performed.

Immunofluorescent staining

PDLSCs were planted with a density of $1 \times 10^{5} \mathrm{cells} / \mathrm{mL}$. After $24 \mathrm{~h}$ of cell attachment, PDLSCs were fixed by $4 \%$ paraformaldehyde was fixed for $1 \mathrm{~h}$. The primary antibody was diluted in proportion with Immunostaining Blocking/Primary Antibody Dilution Buffer (Sangon Biotech) and incubated overnight at $4{ }^{\circ} \mathrm{C}$. PDLSCs were incubated with the corresponding secondary antibody for $2 \mathrm{~h}$ at room temperature. Nuclei were stained with DAPI (1:1000; Santa Cruz) and the cells were mounted on an inverted fluorescent microscope and photographed.

Cell viability assay

PDLSCs were seeded on 96-well plates at a cell density of $1 \times 10^{3}$ cells/well. $0,0.5,1,2,5,10,20$, 50umol/L IGF-1R inhibitor NVP-ADW742 (Beyotime, Shanghai, China) were add into Osteogenic Induction Solution and were used to culture PDLSCs for $24 \mathrm{~h}, 48 \mathrm{~h}, 72 \mathrm{~h}$. Then we washed the wells, added $100 \mathrm{uL} \mathrm{L-}$ DMEM and $10 \mathrm{uL}$ CCK8 (Beyotime, Shanghai, China) per well, and incubated the cells at $37^{\circ} \mathrm{C}$ for $2 \mathrm{~h}$. The optical density was measured down at $450 \mathrm{~nm}$.

Statistical analysis

The experimental data were analyzed by the GraphPad Prism software. All experiments were repeated at least three times, with three replicate wells per design and the data shown as mean \pm standard deviation. 
Statistical significance was assessed by independent sample t test, $p<0.05$ was considered statistically significant.

\section{Results}

\section{High glucose environment inhibits osteogenic differentiation of PDLSCs}

In our experiments, in order to investigate the effect of different glucose concentrations on osteogenic differentiation of PDLSCs, different concentrations of glucose concentration $(5.5 \mathrm{mMol} / \mathrm{L}, 11 \mathrm{mMol} / \mathrm{L}, 25$ $\mathrm{mMol} / \mathrm{L}$ and $44 \mathrm{mMol} / \mathrm{L}$ ) were added to the osteogenic induction solution and stimulated by co-culture with PDLSCs for 21 days, and then analyzed by using alizarin red staining and ALP staining. The results showed that compared with $5.5 \mathrm{Mmol} / \mathrm{L}$ osteogenic induction medium, the amount of mineralized nodule formation of PDLSCs gradually decreased in a concentration dependent manner with the increase of glucose concentration after 21 days of osteogenic induction, and the osteogenic differentiation ability of PDLSCs decreased (Fig. 1a). The osteogenic differentiation transcription factor RUNX2 is a key protein involved in osteoblast differentiation and bone morphogenesis, which is essential for osteoblast maturation as well as intra-membrane and endochondral ossification. SLUG, a transcription factor, is present in stem cells and is essential for osteoblast differentiation. Also, this osteogenic stem cell factor is associated with the transcriptional regulation of stem cell genes and genes activated during osteogenic differentiation. In addition, SLUG also binds to the RUNX2 promoter to promote osteogenic differentiation. The protein expression levels of SLUG as well as the osteogenic differentiation marker RUNX2 were examined by Western Blot after 14 days of osteogenic induction in PDLSCs at different glucose concentrations, the results similarly showed that the expression of the osteogenic marker RUNX2 in PDLSCs at 14 days of osteogenic induction was gradually reduced in a glucose concentrationdependent manner. Meanwhile the protein expression level of SLUG was also gradually reduced (Fig. 1b). This indicated that high glucose significantly inhibited the osteogenic differentiation of PDLSCs. Subsequently, the expression of SLUG, RUNX2 in PDLSCs was examined by RT-qPCR analysis after 7 days of osteogenic differentiation induction by different glucose concentrations. The results similarly showed that the mRNA levels of SLUG, RUNX2 decreased significantly with increasing glucose concentration in the osteogenic induction medium (Fig. 1c). This indicates that high glucose significantly inhibited the osteogenic differentiation of PDLSCs, and the expression level of SLUG was proportional to the osteogenic differentiation of PDLSCs, which suggests that SLUG may be involved in the osteogenic differentiation of PDLSCs.

According to our research results and many existing scientific research literature references, we selected 5. $5 \mathrm{mMol} /$ Land $25 \mathrm{mMol} / \mathrm{L}$ glucose concentration as normal group and high glucose group respectively for follow-up experiments. These results suggest that high glucose environment inhibits the osteogenic differentiation of PDLSCs, and SLUG may be involved in this process.

\section{SUM01 modification of IGF-1R in PDLSCs increased under high glucose environment}


Based on the above results indicating that osteogenic induction medium with different sugar concentrations inhibited osteogenic differentiation of PDLSCs in a concentration-dependent manner, we wanted to find the upstream damage factors affecting osteogenic differentiation of PDLSCs in a high glucose environment. So we selected a concentration of $5.5 \mathrm{mMol} / \mathrm{L}$ for the normal group and 25 $\mathrm{mMol} / \mathrm{L}$ for the high glucose group for the osteogenic induction of PDLSCs for subsequent experiments. Secondly, we wanted to confirm the relationship between SLUG and the decreased osteogenic differentiation ability of PDLSCs under high glucose stimulation, as well as the upstream regulatory factors of SLUG. The activation of IGF-1R regulates and maintains many key signaling pathways of cell homeostasis, including cell survival, growth, proliferation and differentiation. The imbalance of sumoylation is related to different disease. Meanwhile, SUMO1 is related to nuclear translocation and accumulation of IGF-1R. Compared with the normal group, the protein expression levels of IGF-1R and SUM01 in PDLSCs induced by high glucose were significantly increased after 7 days, indicating that the degree of sumoylation in PDLSCs was also enhanced (Fig. 2a). In cell models of other diseases, IGF-1R and SUMO1 have been studied thoroughly. However, the osteogenic differentiation ability of stem cells has not been studied, especially in the etiology of diabetic osteoporosis. Recent studies have shown that sumoylation occurs in IGF-1R, which induces nuclear accumulation of IGF-1R receptor [21]. Therefore, we speculate that high glucose stimulation may promote the sumoylation of IGF-1R on cell membrane. SUM01 modification occurs at three evolutionarily conservative lysine residues of the $\beta$-subunit of IGF1R. Therefore, after 7 days of osteogenic induction culture of PDLSCs, we used anti-IGF-1R for immunoprecipitation followed by detection of SUMO1 by immunoblotting. The results showed that the expression in the bands of SUMO1 bound to IGF-1R was significantly richer in the high glucose group than in the normal group (Fig. 2b). Further Western Blot analysis showed that sumoylation of IGF-1R was detected on the protein. Interestingly, it was found that the imprinting of IGF-1R had obvious tailing phenomenon (Fig. 2C). Taken together, these data indicated that sumoylation of IGF-1R were induced by high glucose in PDLSCs.

\section{Sumoylation of IGF1R inhibits osteogenic differentiation of PDLSCs by binding to SLUG through nucleus entry}

As mentioned above, the expression of IGF-1R in PDLSCs stimulated by high glucose was increased, and the degree of sumoylation was also enhanced. In order to explore whether the change of IGF-1R induced by high glucose is the key factor affecting the osteogenic differentiation ability of PDLSCs, we isolated and extracted the membrane protein and nuclear protein. Western blotting showed that the expression of IGF-1R in both membrane protein and nuclear protein increased in PDLSCs cultured with high glucose, but the expression of SLUG in nucleus decreased (Fig. 3a). This suggests to us that sumoylation of IGF$1 R$ may enter the nucleus and bind to SLUG, which coincides with the inhibition of osteogenic differentiation of PDLSCs in high glucose environment. To further assess the effect of high glucose on IGF-1R nuclear aggregation following with intranuclear SLUG, we performed cellular immunofluorescence staining for IGF-1R and SLUG. We found elevated IGF-1R expression in the nuclei of PDLSCs cultured with 
high glucose and decreased expression of SLUG in the nucleus, which is consistent with our previous immunoblot analysis. More interestingly, we found increased nuclear localization of IGF-1R with SLUG in the nucleus of PDLSCs cultured in high glucose (Fig. 3b). To further verify the interaction of IGF-1R with SLUG in the high glucose situation, we performed immunoprecipitation with anti-IGF-1R antibody after the cells were cultured separately for 7 days. First, we analyzed the binding of IGF-1R to SLUG by immunoprecipitation, and again found that the binding of IGF-1R to SLUG was significantly increased by high glucose stimulation (Fig. 3b). The expression of IGF-1R was also promoted at the target molecular weight, which was consistent with the previous experimental results obtained (Fig. 2c). From these data, it was shown that high glucose stimulated sumoylation of IGF-1R within PDLSCs and that binding to SLUG after nucleation inhibited the osteogenic differentiation ability of the cells.

\section{IGF-1R inhibitor reverses the inhibition of osteogenic differentiation induced by high glucose}

To further demonstrate whether IGF-1R is a key factor in the inhibition of osteogenic differentiation of PDLSCs by high glucose environment, we then used IGF-1R inhibitor NVP-ADW742 to block the downstream signaling pathway in the subsequent experiments. In recent years, NVP-ADW742 has been used as IGF-1R inhibitor in many studies. Firstly, to clarify the effect of NVP-ADW742 concentration on the toxicity and proliferation of PDLSCs, we performed CCK-8 assay in 96-well plates. PDLSCs were seeded in a $96-$ well plate at density of $1 \times 10^{6} \mathrm{cells} / \mathrm{ml}$. The following day, medium was changed in DMEM plus 10\% FBS with NVP-ADW742 (0 50 umol/L). Cells were collected after 24h, 48h and 72h. CCK-8 assay prompted us to select a concentration of $5 \mathrm{umol} / \mathrm{L}$ NVP-ADW742 for subsequent experiments (Fig. 4a). After stimulation with 5 umol/L NVP-ADW742 for $72 \mathrm{~h}$, the expression level of IGF-1R in PDLSCs was detected, and it was found that the expression level of IGF-1R was indeed decreased (Fig. 4b). Then after the medium of NVP-ADW742 group was changed to high glycogenic osteogenic induction medium, the culture was continued until 7 days after Western Blotting, and it was found that the expression level of RUNX2,SLUG protein in NVP-ADW742 group was slightly higher than that in high glucose osteogenic induction group (Fig. 4b). Similarly, the medium of NVP-ADW742 group was replaced with high glucose osteogenic induction medium and the culture was continued until 14 days after alizarin red staining and ALP staining, and the mineralization results were consistent with the Western blotting assay in Fig. $4 \mathrm{~b}$ (Fig. 4c). These findings suggest to us that IGF-1R inhibitor NVP-ADW742 alleviated the inhibition of osteogenic differentiation caused by high glucose, but the osteogenic mineralization capacity was still inferior to that of PDLSCs cultured in the normal glucose concentration group. Thus, it seems that the high expression of IGF-1R is indeed a key factor leading to the inhibition of osteogenic differentiation in PDLSCs. Genetic studies have shown that the IGF-1R/PI3K/AKT signal transduction pathway is involved in a variety of animals, including nematodes, Drosophila and mammals, and is closely linked to the development of many diseases[22]. Recently PI3K/AKT and its downstream signaling pathways have received increasing attention in tumor and osteogenic differentiation. To investigate the downstream signaling pathway of IGF-1R inhibiting osteogenic differentiation of PDLSCs, we performed Western blotting assay on three groups of proteins and found that IGF-1R activated PI3K/AKT signaling pathway and the activation degree of PI3K, pAKT was positively correlated with IGF-1R (Fig. 4D). We then highly 
suspected that the PI3K/AKT signaling pathway downstream of IGF-1R plays the same role in the high glucose environment to inhibit osteogenic differentiation of PDLSCs.

\section{Discussion}

The key point of this paper is to find a new mechanism of high glucose stimulation inducing IGF-1R nuclear transport on PDLSCs membrane, thereby inhibiting the osteogenic differentiation of PDLSCs (Fig. 5a). IGF-1R nuclear transfer has been fully studied in many other fields, such as oncology and ophthalmology [23]. Our research further shows that the nuclear transfer of IGF-1R in high glucose environment is mediated by SUMO protein modification. More interestingly, sumoylation of IGF-1R nuclear translocation binds to SLUG in the nucleus and thus inhibits downstream osteogenic related proteins (Fig. 5b). This has never been studied in the aspect of stem cell osteogenesis differentiation. This finding may provide an effective method for the treatment of periodontal disease in diabetic patients.

Bone loss and bone remodeling around adult teeth are regulated by a dynamic balance between osteoclasts and osteoblasts. However, under the influence of glucose, aging, and inflammation, the expression level of the osteogenesis-related marker protein RUNX2 decreases, leading to difficulties in alveolar bone regeneration. Diabetes and periodontitis have been considered to be biologically related and high glucose induces oxidative stress, which inhibits proliferation and osteogenic differentiation of PDLSCs [24]. It has also been found that high glucose induces NF-KB activation and leads to high expression of IL- 6 and IL-8, thereby inhibiting osteogenic differentiation of PDLSCs [25]. The osteogenic differentiation ability of PDLSCs was significantly reduced in the high glucose state, and these findings are consistent with the findings of our group.

Previous studies have shown that there are multiple signaling pathways involved in the regulation of osteoblast metabolic homeostasis during osteogenic differentiation, such as the PI3K/AKT signaling pathway that promotes the progression of osteoporosis [26]. IGF-1R is a membrane protein that is expressed on the surface of a variety of cells, including hepatocytes, myocytes and osteocytes [27]. Recent studies have shown that IGF-1R can be involved in alterations in blood glucose levels and bone metabolism $[28,29]$ and interferes with the osteogenesis-related Wnt/ $\beta$-catenin signaling pathway $[30$, 31]. It has also been shown in animal experiments that IGF-1R expression levels and phosphorylation levels of IGF-1R are also significantly higher in OVX + streptozotocin-induced osteoporosis model rats than in normal rats [26]. Sumoylation of proteins is an important regulatory element regulating protein function in the human body, which is involved in the pathogenesis of a variety of human diseases. More and more evidence shows that sumoylation plays a very important role in the pathogenesis of diabetes [32]. As we all know, osteoporosis is a serious complication of diabetes, which has been the focus of scholars for a long time. However, we unexpectedly found that sumoylation of IGF-1R plays a significant role in the process of stem cell osteogenic differentiation, especially in regulating downstream osteogenic-related transcription factors such as SLUG and RUNX2. SLUG is a zinc-finger transcription factor that has long been a focus of research in directing epithelial mesenchymal transition associated with the progression of tumor diseases. However, recent studies have shown that this EMT-related 
transcription factor also regulates the self-renewal and osteogenic differentiation potential of bone marrow mesenchymal stem cells [33]. Secondly, we also significantly reversed the inhibition of osteogenic differentiation caused by the high glucose environment after adding appropriate amounts of IGF-1R inhibitors to PDLSCs in osteogenic differentiation culture. Thus IGF-1R and its downstream signaling axis may play a role in inhibiting osteogenic differentiation of stem cells, as similarly demonstrated in our group's study. However, the role of post-translational modification of IGF-1R protein in high glucose environment deserves further investigation, and the protein modification makes IGF-1R structure more complex and function more perfect.

\section{Conclusion}

In summary, our data demonstrated that IGF-1R inhibits the osteogenic differentiation of PDLSCs via combining with SLUG stimulated by high glucose. Therefore, IGF-1R might be a molecular target to regulate the osteogenic differentiation for therapeutic agents in dental medicine.

\section{Declarations}

\section{Acknowledgements}

Not applicable.

\section{Authors' contributions}

QZ and XF conceived and designed the experiments. RJ, MW, XS, SH, ZX, and JP, LL, CJ performed the experiments. RJ and XF analyzed the data. RJ, XS and MW wrote the paper. All authors read and approved the final manuscript.

\section{Funding}

The study was supported by Nantong City Science and Technology Projects Funds (MS12020030).

\section{Availability of data and materials}

The datasets used and/or analyzed during the current study are available from the corresponding author on reasonable request.

\section{Ethics approval and consent to participate}

The study was approved by the Ethics Committee of the Affiliated Hospital of Nantong University.

\section{Consent for publication}

Not applicable. 


\section{Competing interests}

The authors declare that they have no competing interests.

\section{References}

1. Cho NH, Shaw JE, Karuranga S, Huang Y, da Rocha Fernandes JD, Ohlrogge AW, et al. IDF Diabetes Atlas: Global estimates of diabetes prevalence for 2017 and projections for 2045. Diabetes Res Clin Pract. 2018;138:271-81.

2. Hajishengallis G. Periodontitis: from microbial immune subversion to systemic inflammation. Nat Rev Immunol. 2015;15(1):30-44.

3. Darveau RP. Periodontitis: a polymicrobial disruption of host homeostasis. Nat Rev Microbiol. 2010;8(7):481-90.

4. Pritchard JM, Giangregorio LM, Atkinson SA, Beattie KA, Inglis D, loannidis G, et al. Association of larger holes in the trabecular bone at the distal radius in postmenopausal women with type 2 diabetes mellitus compared to controls. Arthritis Care Res (Hoboken). 2012;64(1):83-91.

5. Xia Y, Sun J, Zhao L, Zhang F, Liang XJ, Guo Y, et al. Magnetic field and nano-scaffolds with stem cells to enhance bone regeneration. Biomaterials. 2018;183:151-70.

6. Chen XJ, Shen YS, He MC, Yang F, Yang P, Pang FX, et al. Polydatin promotes the osteogenic differentiation of human bone mesenchymal stem cells by activating the BMP2-Wnt/beta-catenin signaling pathway. Biomed Pharmacother. 2019;112:108746.

7. Ma Y, Qi M, An Y, Zhang L, Yang R, Doro DH, et al. Autophagy controls mesenchymal stem cell properties and senescence during bone aging. Aging Cell. 2018;17(1).

8. Gu Z, Tan W, Ji J, Feng G, Meng Y, Da Z, et al. Rapamycin reverses the senescent phenotype and improves immunoregulation of mesenchymal stem cells from MRL/lpr mice and systemic lupus erythematosus patients through inhibition of the mTOR signaling pathway. Aging (Albany NY). 2016;8(5):1102-14.

9. Dong R, Yu J, Yu F, Yang S, Qian Q, Zha Y. IGF-1/IGF-1R blockade ameliorates diabetic kidney disease through normalizing Snail1 expression in a mouse model. Am J Physiol Endocrinol Metab. 2019;317(4):E686-E98.

10. Li J, Choi E, Yu H, Bai XC. Structural basis of the activation of type 1 insulin-like growth factor receptor. Nat Commun. 2019;10(1):4567.

11. Yang $Y, H e Y$, Wang $X$, Liang Z, He G, Zhang P, et al. Protein SUMOylation modification and its associations with disease. Open Biol. 2017;7(10).

12. Huang W, Liang Y, Dong J, Zhou L, Gao C, Jiang C, et al. SUMO E3 Ligase PIASy Mediates High Glucose-Induced Activation of NF-kappaB Inflammatory Signaling in Rat Mesangial Cells. Mediators Inflamm. 2017;2017:1685194. 
13. Warsito D, Lin Y, Gnirck AC, Sehat B, Larsson O. Nuclearly translocated insulin-like growth factor 1 receptor phosphorylates histone $\mathrm{H} 3$ at tyrosine 41 and induces SNAI2 expression via Brg1 chromatin remodeling protein. Oncotarget. 2016;7(27):42288-302.

14. Titone R, Zhu M, Robertson DM. Mutual regulation between IGF-1R and IGFBP-3 in human corneal epithelial cells. J Cell Physiol. 2019;234(2):1426-41.

15. Rauch A, Haakonsson AK, Madsen JGS, Larsen M, Forss I, Madsen MR, et al. Osteogenesis depends on commissioning of a network of stem cell transcription factors that act as repressors of adipogenesis. Nat Genet. 2019;51(4):716-27.

16. Torreggiani E, Lisignoli G, Manferdini C, Lambertini E, Penolazzi L, Vecchiatini R, et al. Role of Slug transcription factor in human mesenchymal stem cells. J Cell Mol Med. 2012;16(4):740-51.

17. Tang Y, Feinberg T, Keller ET, Li XY, Weiss SJ. Snail/Slug binding interactions with YAP/TAZ control skeletal stem cell self-renewal and differentiation. Nat Cell Biol. 2016;18(9):917-29.

18. Zheng Y, Dong C, Yang J, Jin Y, Zheng W, Zhou Q, et al. Exosomal microRNA-155-5p from PDLSCs regulated Th17/Treg balance by targeting sirtuin-1 in chronic periodontitis. J Cell Physiol. 2019;234(11):20662-74.

19. Bao L, Zhang X, Xu Y, Wang M, Song Y, Gu Y, et al. Dysfunction of MiR-148a-NRP1 Functional Axis Suppresses Osteogenic Differentiation of Periodontal Ligament Stem Cells Under Inflammatory Microenvironment. Cell Reprogram. 2019;21(6):314-22.

20. Zhang J, Lu X, Feng G, Gu Z, Sun Y, Bao G, et al. Chitosan scaffolds induce human dental pulp stem cells to neural differentiation: potential roles for spinal cord injury therapy. Cell Tissue Res. 2016;366(1):129-42.

21. Deng $H$, Lin $Y$, Badin M, Vasilcanu D, Stromberg T, Jernberg-Wiklund $H$, et al. Over-accumulation of nuclear IGF-1 receptor in tumor cells requires elevated expression of the receptor and the SUMOconjugating enzyme Ubc9. Biochem Biophys Res Commun. 2011;404(2):667-71.

22. Sergi C, Shen F, Liu SM. Insulin/IGF-1R, SIRT1, and FOXOs Pathways-An Intriguing Interaction Platform for Bone and Osteosarcoma. Front Endocrinol (Lausanne). 2019;10:93.

23. Codony-Servat J, Cuatrecasas M, Asensio E, Montironi C, Martinez-Cardus A, Marin-Aguilera M, et al. Nuclear IGF-1R predicts chemotherapy and targeted therapy resistance in metastatic colorectal cancer. Br J Cancer. 2017;117(12):1777-86.

24. Zheng DH, Han ZQ, Wang XX, Ma D, Zhang J. Erythropoietin attenuates high glucose-induced oxidative stress and inhibition of osteogenic differentiation in periodontal ligament stem cell (PDLSCs). Chem-Biol Interact. 2019;305:40-7.

25. Kato H, Taguchi Y, Tominaga K, Kimura D, Yamawaki I, Noguchi M, et al. High Glucose Concentrations Suppress the Proliferation of Human Periodontal Ligament Stem Cells and Their Differentiation Into Osteoblasts. J Periodontol. 2016;87(4):e44-51.

26. Zhang Z, Ren H, Shen G, Zhao W, Shang Q, Yu X, et al. IGF-1R/beta-catenin signaling axis is implicated in streptozotocin exacerbating bone impairment in ovariectomized rats. Climacteric. 2021;24(2):179-86. 
27. Cheng Y, Liu S, Zhang X, Wu Q, Li S, Fu H, et al. Expression Profiles of IGF-1R Gene and Polymorphisms of its Regulatory Regions in Different Pig Breeds. Protein J. 2016;35(3):231-6.

28. Solomon-Zemler R, Basel-Vanagaite L, Steier D, Yakar S, Mel E, Phillip M, et al. A novel heterozygous IGF-1 receptor mutation associated with hypoglycemia. Endocr Connect. 2017;6(6):395-403.

29. Pelosi P, Lapi E, Cavalli L, Verrotti A, Pantaleo M, de Martino M, et al. Bone Status in a Patient with Insulin-Like Growth Factor-1 Receptor Deletion Syndrome: Bone Quality and Structure Evaluation Using Dual-Energy X-Ray Absorptiometry, Peripheral Quantitative Computed Tomography, and Quantitative Ultrasonography. Front Endocrinol (Lausanne). 2017;8:227.

30. Palsgaard J, Emanuelli B, Winnay JN, Sumara G, Karsenty G, Kahn CR. Cross-talk between insulin and Wnt signaling in preadipocytes: role of Wnt co-receptor low density lipoprotein receptor-related protein-5 (LRP5). J Biol Chem. 2012;287(15):12016-26.

31. Rota LM, Wood TL. Crosstalk of the Insulin-Like Growth Factor Receptor with the Wnt Signaling Pathway in Breast Cancer. Front Endocrinol (Lausanne). 2015;6:92.

32. Yang Z, Zhang Y, Sun S. Deciphering the SUMO code in the kidney. J Cell Mol Med. 2019;23(2):711-9.

33. Tang Y, Weiss SJ. Snail/Slug-YAP/TAZ complexes cooperatively regulate mesenchymal stem cell function and bone formation. Cell Cycle. 2017;16(5):399-405.

\section{Figures}


a

$5.5 \mathrm{mMol} / \mathrm{L} \quad 11 \mathrm{mMol} / \mathrm{L} \quad 25 \mathrm{mMol} / \mathrm{L} \quad 44 \mathrm{mMol} / \mathrm{L}$
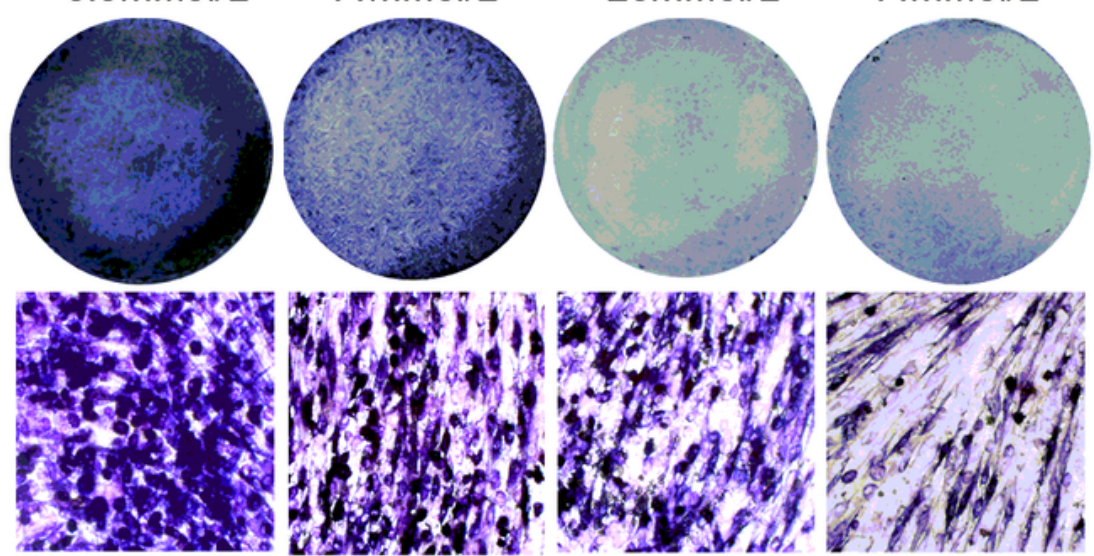

ALP staining
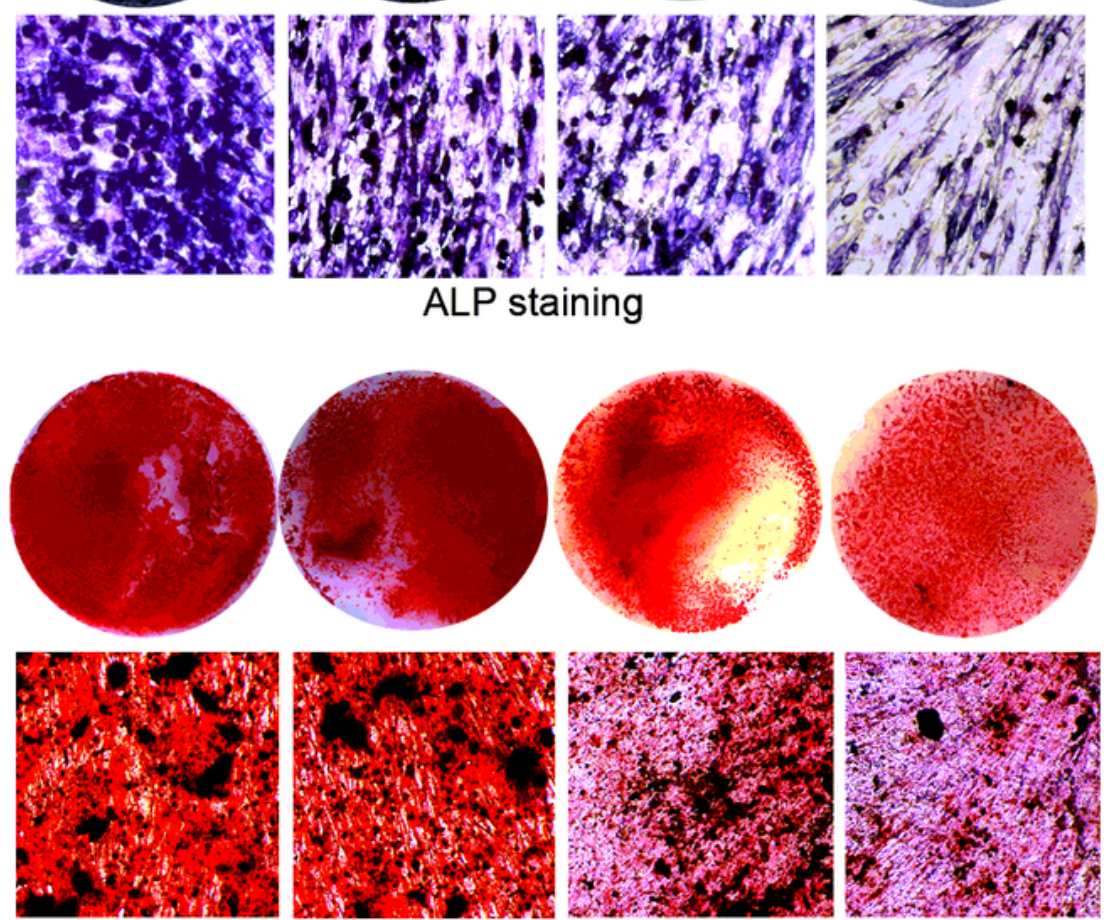

Alizarin red staining

C

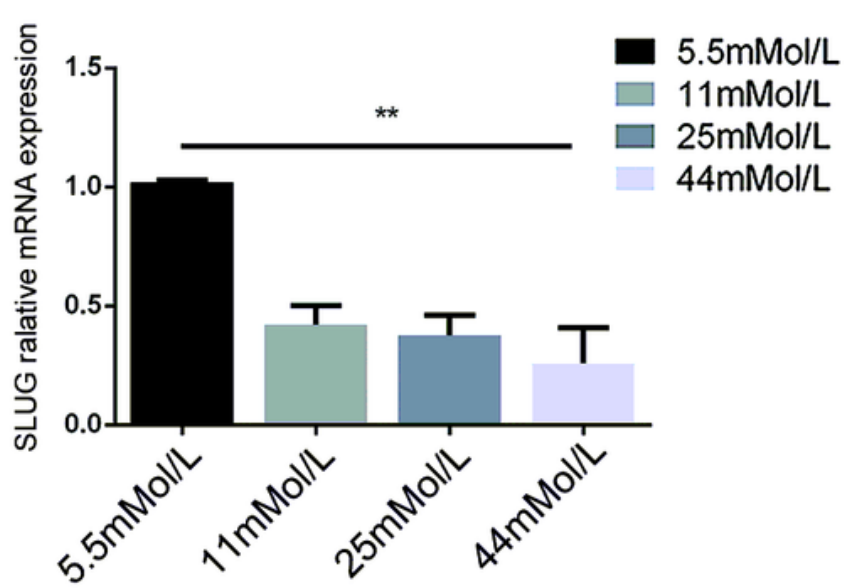

b
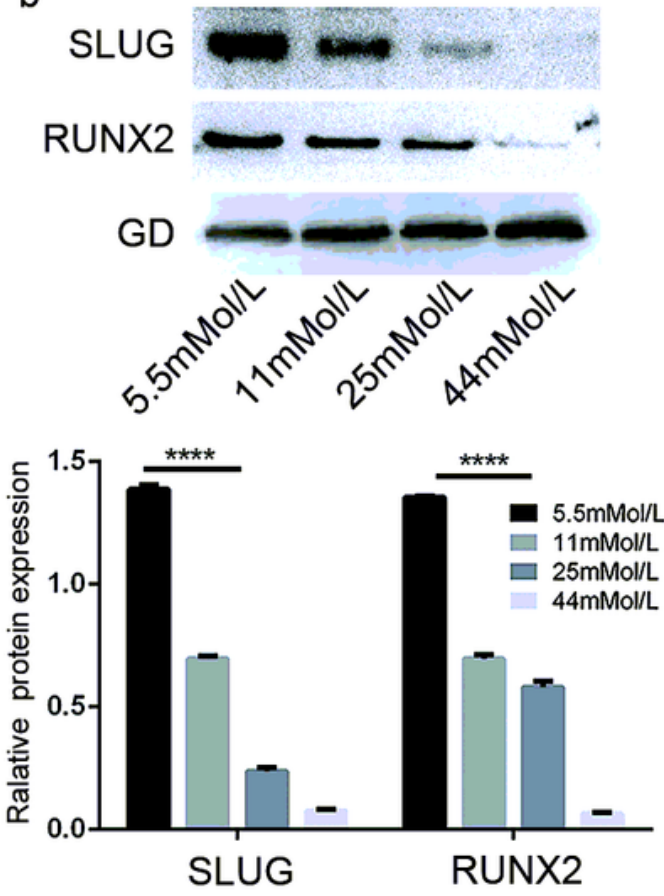

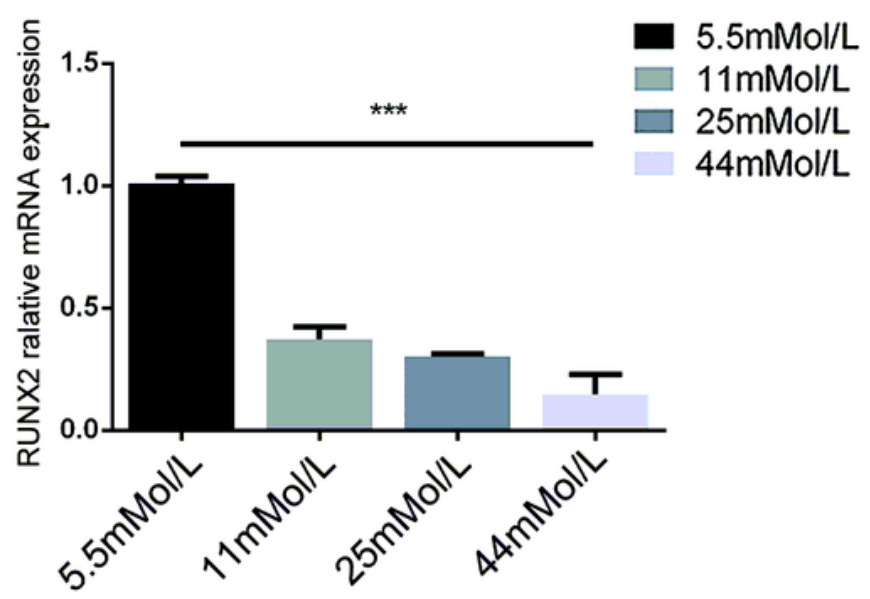

Figure 1

Osteogenic differentiation of PDLSCs under osteogenic differentiation induction solution with different glucose concentrations. PDLSCs were cultured in an osteogenic medium containing glucose at concentrations of $5.5 \mathrm{mMol} / \mathrm{L}, 11 \mathrm{mMol} / \mathrm{L}, 25 \mathrm{mMol} / \mathrm{L}, 44 \mathrm{mMol} / \mathrm{L}$. (a) Osteogenic differentiation was determined by alizarin red staining and ALP staining after 21 days; (b) RUNX2 and SLUG was measured by Western Blot at 14 days of osteogenic induction. GAPDH as an internal reference. And Quantitative 
analysis of expression levels ( $\left.{ }^{*} \mathrm{P}<0.05\right)$; (c) The levels of RUNX2 and SLUG in PDLSCs were evaluated by RT-qPCR at 7 days of osteogenic induction. The data are expressed as the mean \pm SD of at least three replicates, ${ }^{*} p<0.05 ;{ }^{*} p<0.01 ; * \star * p<0.001$, all results were obtained from at least three independent experiments. PDLSCs, periodontal ligament stem cells; RT-qPCR, real-time quantitative polymerase chain reaction; SD, standard deviation.

a
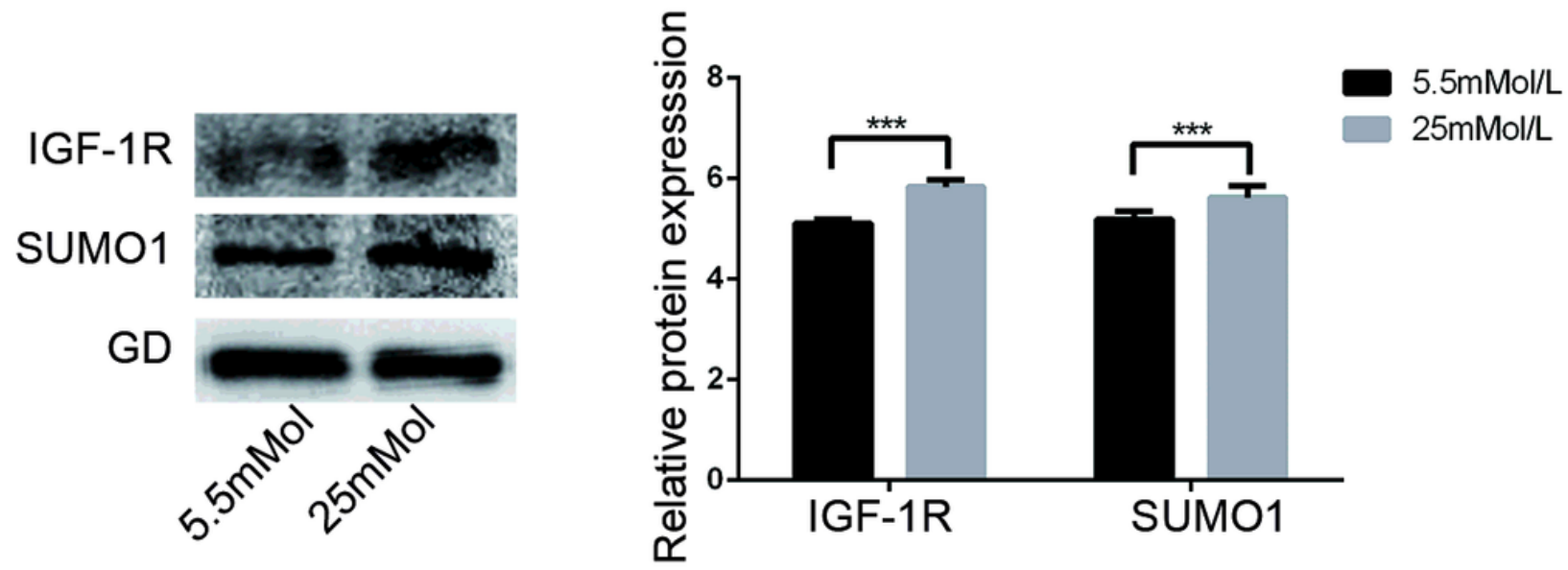

b

C

IP:IGF-1R

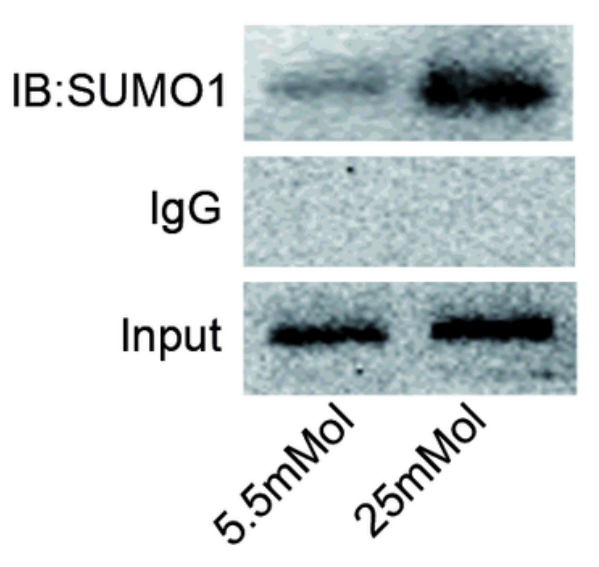

IP Input

sumoylation of IGF1R

Re-blot:

IGF1R

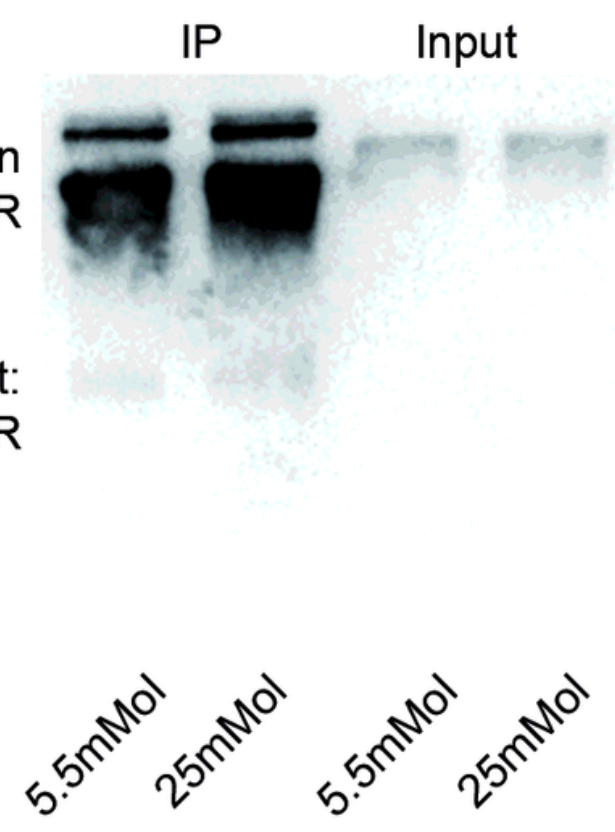

\section{Figure 2}

High glucose promoted sumoylation of IGF-1R. $5.5 \mathrm{mMol} / \mathrm{L}$ and $25 \mathrm{mMol} / \mathrm{L}$ groups were selected for follow-up experiment. (a) IGF-1R and SUM01 was measured by Western Blot at 7 days of osteogenic induction. GAPDH as an internal reference. And Quantitative analysis of expression levels $\left({ }^{*} P<0.05\right)$. (b) 
Sumoylation of IGF-1R was detected by immunoprecipitation (IP) with anti-IGF-1R antibody followed by Western blotting with anti-SUMO1 antibody. IGF-1R was conjugated with SUM01 in PDLSCs. (c) SUMOylation of IGF-1R were determined by IP of IGF-1R and IB for IGF-1R. The data are expressed as the mean $\pm S D$ of at least three replicates, ${ }^{\star} p<0.05 ;{ }^{* \star} p<0.01 ;{ }^{* \star} p<0.001$, all results were obtained from at least three independent experiments.

a

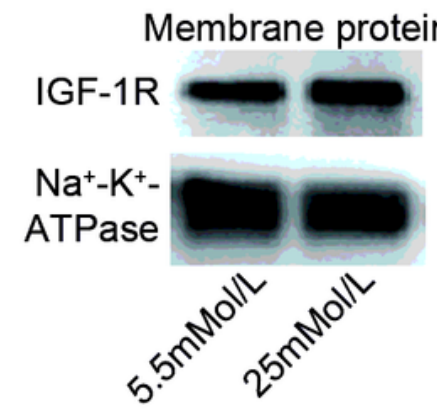

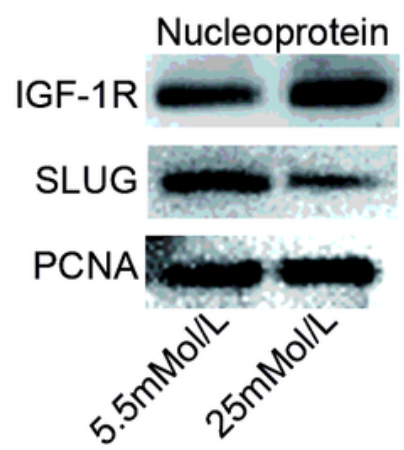

b

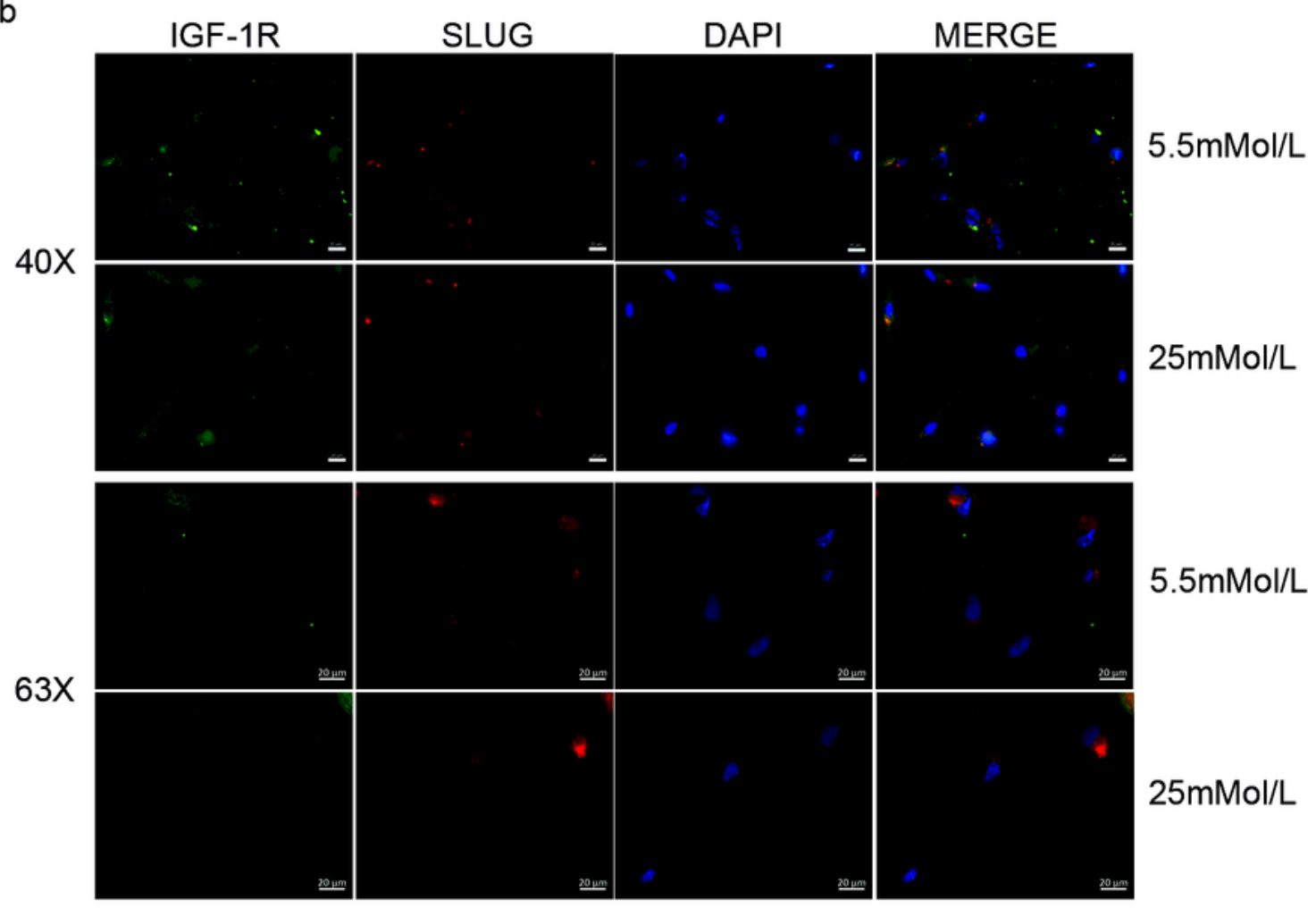

C

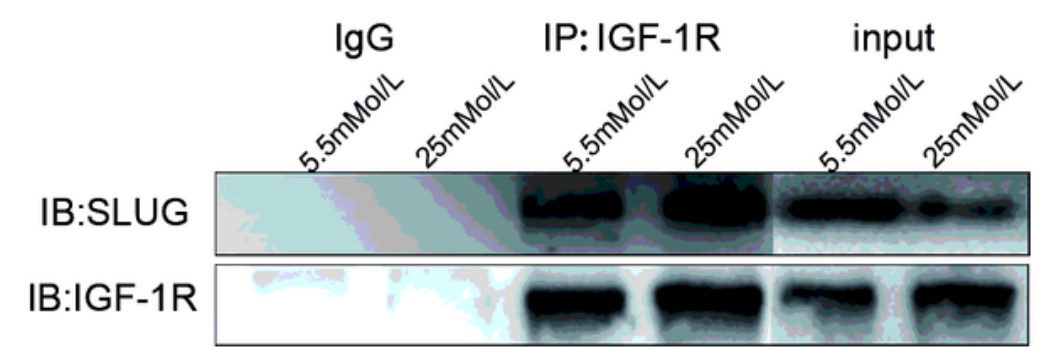

Figure 3 
Sumoylation of IGF1R inhibits osteogenic differentiation of PDLSCs. (a) the expression levels of IGF-1R and SLUG were detected by Western Blot in membrane protein and nuclear protein after 7 days of osteogenic induction. Na+-K+- ATPase and PCNA as an internal reference. (b) The expression of IGF-1R (green) and SLUG (red) in PDLSCs were examined by immunofluorescent staining. Nuclei are stained with DAPI (blue). Magnification: $\times 40$ and $\times 63$. (c) IGF-1R was conjugated with SLUG in PDLSCs was detected by immunoprecipitation (IP) with anti-IGF-1R antibody followed by Western blotting with anti-SLUG antibody. All results were obtained from at least three independent experiments.

a CCK-8 assay

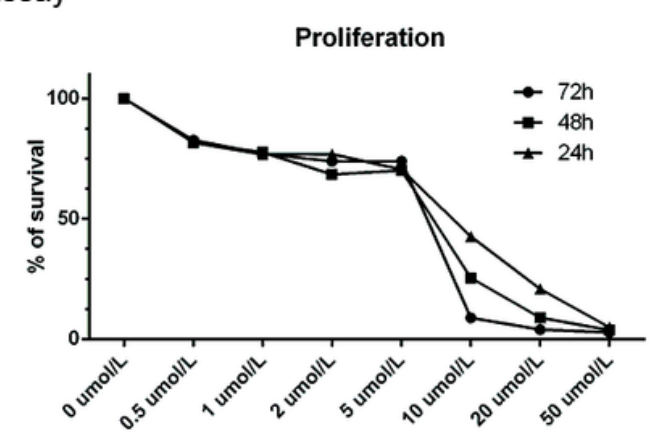

C

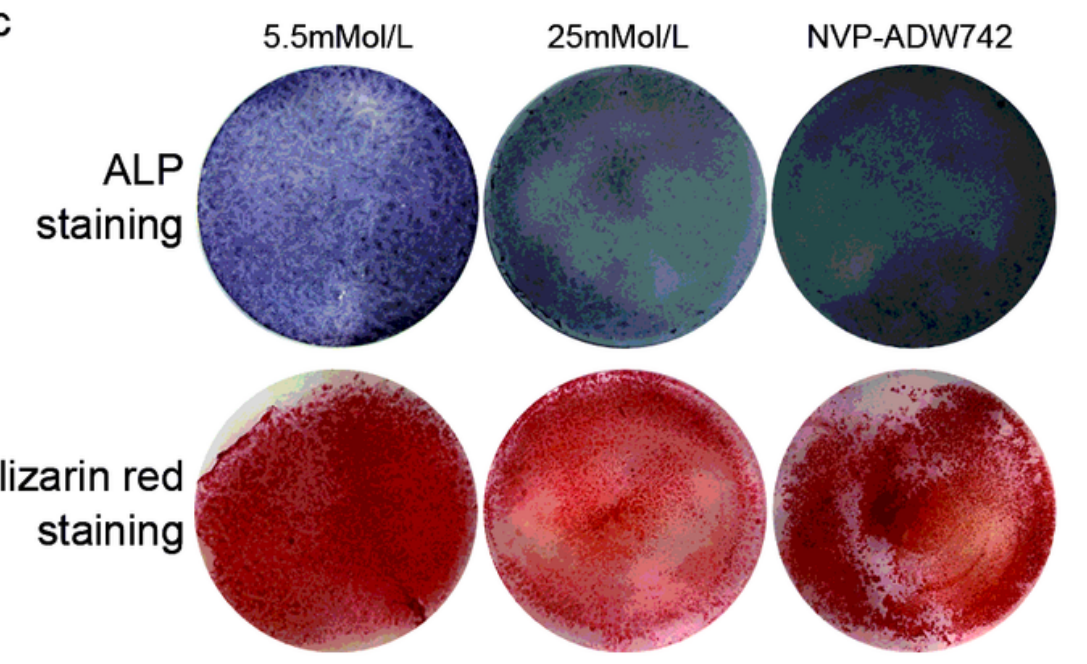

b

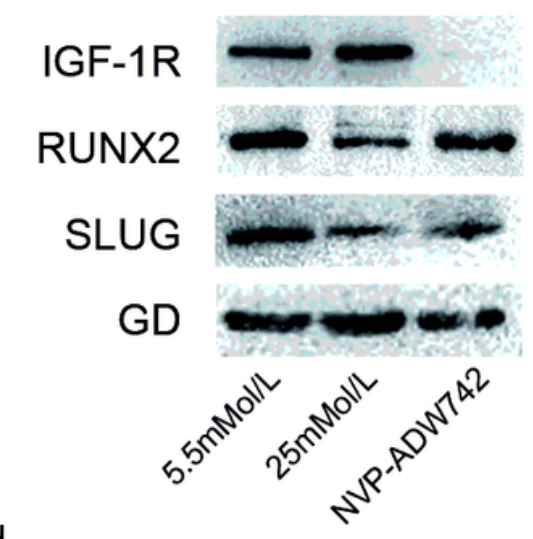

d
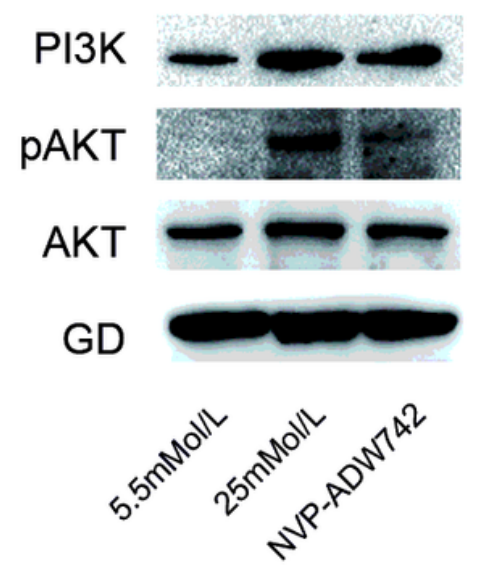

\section{Figure 4}

Inhibition of IGF-1R in high glucose environment promotes osteogenic differentiation of PDLSCs. (a) the effects of NVP-ADW742 treatment on proliferation were assayed by CCK-8 assay. Cells were cultured for $24 \mathrm{~h}, 48 \mathrm{~h}$, and $72 \mathrm{~h}$ in the indicated concentions of NVP-ADW742, from 0 to $50 \mathrm{umol} / \mathrm{L}$. The results were obtained from at least three independent experiments. (b) IGF-1R, RUNX2 and SLUG was measured by Western Blot at 7 days of osteogenic induction. NVP-ADW742 group, NVP-ADW742 was changed to high glucose osteogenic induction medium after $72 \mathrm{~h}$ stimulation and continued to culture until 7 days. GAPDH as an internal reference. (c) Osteogenic differentiation was determined by alizarin red staining and ALP staining after 21 days. NVP-ADW742 group, NVP-ADW742 was changed to high glucose osteogenic induction medium after $72 \mathrm{~h}$ stimulation and continued to culture until 14 days. (d) Western Blot was 
performed and found that NVP-ADW742 selectively inhibits the PI3K/AKT signing pathway in protein level, which is consistent with the expression level of IGF-1R. All results were obtained from at least three independent experiments. PI3K, phosphatidylinositol-3-kinase; AKT, protein kinase B.

a

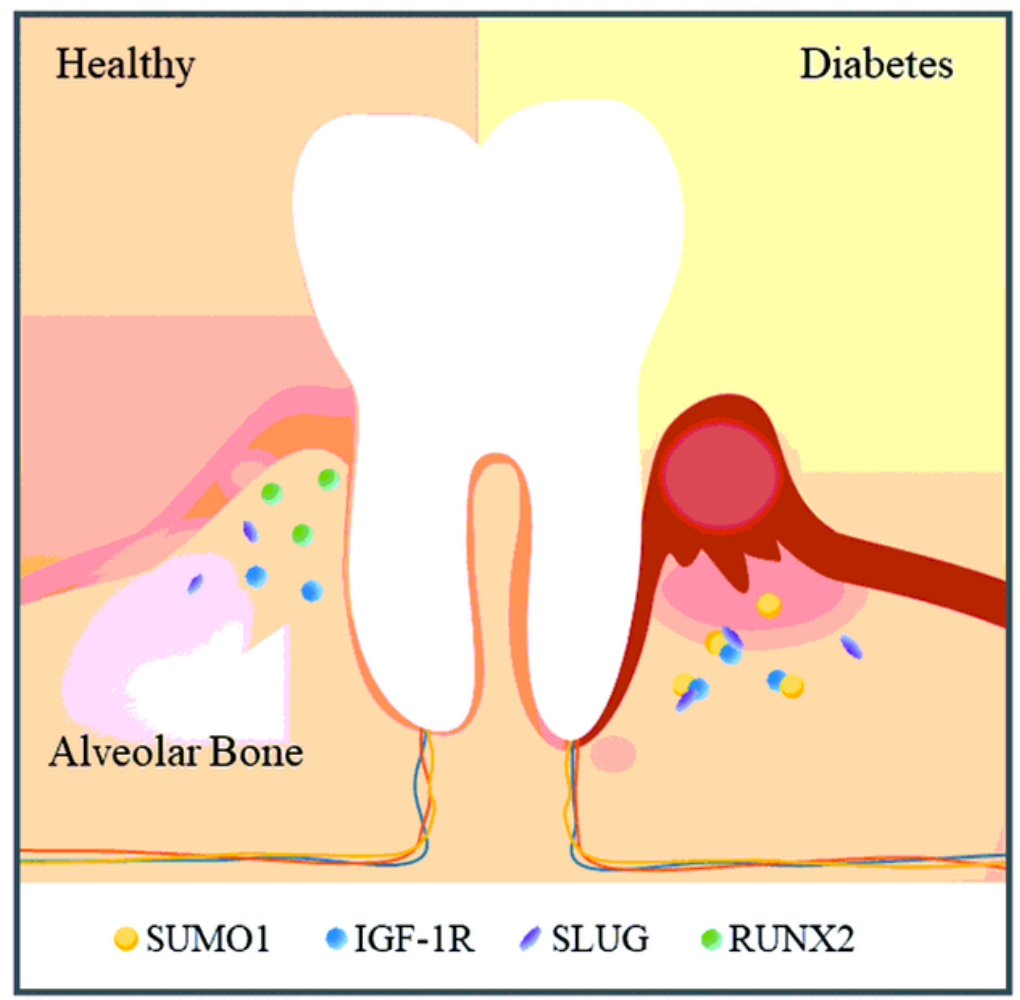

b

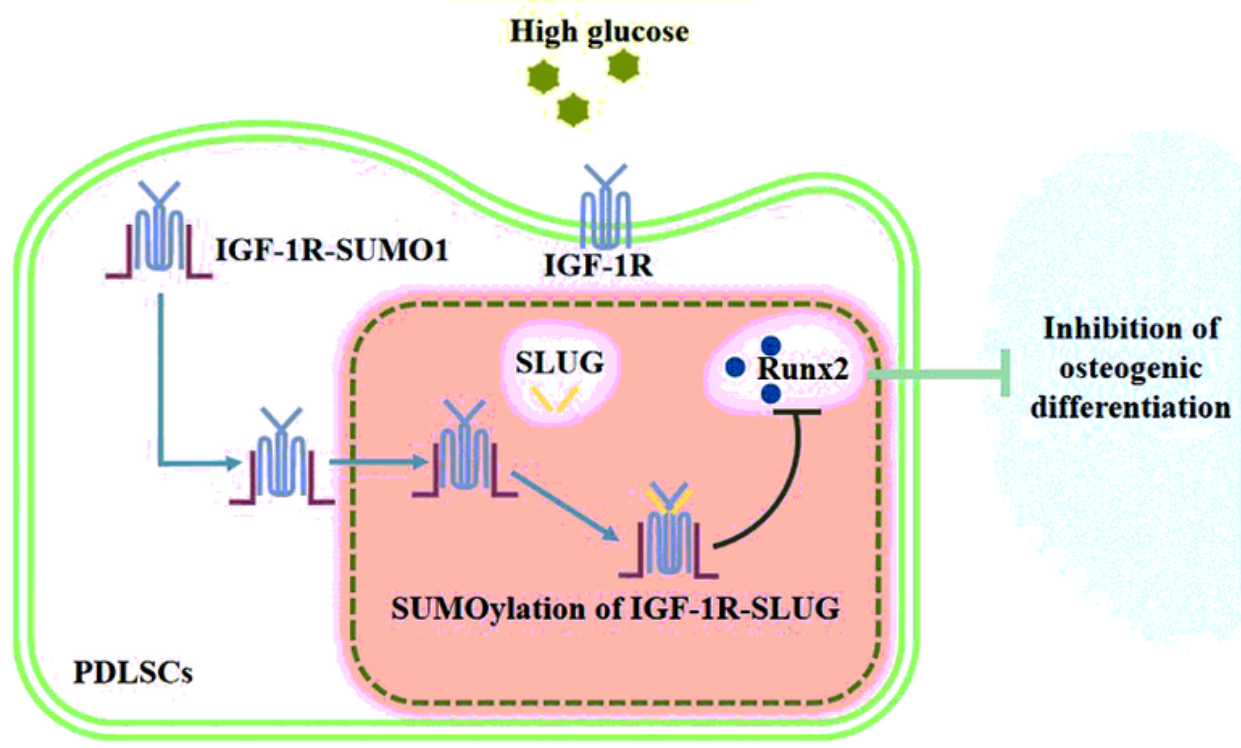

Figure 5

The mechanism of IGF-1R inhibiting osteogenic differentiation of PDLSCs by SUMO modification and binding to SLUG in the nucleus under high glucose stimulation. Under normal conditions, the regenerative function of the alveolar bone around the teeth is stable and PDLSCs can differentiate into osteoblasts 
and other multifaceted differentiation, whereas in diabetic patients, the alveolar bone is continuously resorbed and the teeth are loosened, and IGF-1R is overexpressed. (a) In this study, osteogenic cultures with high glucose concentration were used to culture PDLSCs to mimic the periodontal tissue microenvironment of diabetic patients. Under the stimulation of high glucose, the expression level of IGF$1 \mathrm{R}$ located on the cell membrane was significantly increased, along with the enhancement of SUMO modifications. Subsequently, sumolylation of IGF-1R entered the nucleus to bind to SLUG in the nucleus to inhibit downstream osteogenic-related proteins, resulting in reduced osteogenic differentiation of PDLSCs under high glucose environment. (b) IGF-1R: The insulin-like growth factor 1 receptor; SUMO1: Small Ubiquitin Like Modifier 1; SLUG: Snail Family Transcriptional Repressor 2; RUNX2: RUNX Family Transcription Factor 2; PDLSCs: Periodontal membrane stem cells. 